\title{
ARTICLE
}

\section{Application of the Charge to Time Conversion Technique in Radiation Spectroscopy}

\author{
Yanchang $\operatorname{LIN}^{1,2^{*}}$ \\ ${ }^{1}$ Department of Engineering Physics, Tsinghua University, Beijing, China \\ ${ }^{2}$ College of Nuclear Technology and Automation Engineering, Chengdu University of Technology, Chengdu, China
}

\begin{abstract}
A Charge to Time Conversion (QTC) technique is proposed to provide the energy spectrum measurement in radiation spectroscopy. Since the width of QTC logical output signal is proportional to the input charge, it can be easily converted into a digital value in the programmable logic device FPGA or CPLD. As a pulse area sensitive circuit, QTC is expected to have better sensitivity, higher noise rejection rate and less waveform dependency, with simpler circuit configuration, lower cost and higher reliability. Interfacing to scintillation detectors with Photo Multiplier Tube (PMT) for a measurement of gamma energy spectrum needs neither pulse shaping nor baseline restoration circuit. The input stage of the QTC can be configured to be current sensitive and connect to a Silicon Surface Barrier (SSB) detector without charge sensitive preamplifier. This circuit configuration was implemented and used in an alpha spectrum based radon measurement. The application circuits and some test results are presented in the paper.
\end{abstract}

\section{KEYWORDS: Charge to Time Conversion, Multi-Channel Analyzer, scintillation detector, gamma spectrum, Silicon Surface Barrier detector, alpha spectrum}

\section{Introduction}

Pulse height Multi-Channel Analyzer (MCA) is generally performed by a pulse peak holding circuit followed by an ADC. In this scheme, the input pulse height in proportion to the energy deposit in the detector will be sampled. The peak holding circuit is sensitive to any sharp peak as well as ripple noise. Analog shaping circuit for converting the input pulse into a quasi-Gauss shape is normally adopted ${ }^{1)}$. In mass production of such an analog signal processing circuit, maintaining precision and consistency in all the products are always challenges.

In recent years, with the development of electronic techniques, such as flash ADC, FPGA and DSP, it is already possible to digitalize the waveform of a fast input pulse from a radiation detector and pick up energy information by DSP mode at the meantime ${ }^{2), 3,4)}$. Shaping function can also be performed by the DSP method instead of the analog circuit. However, the signal to noise ratio (SNR) for a flash ADC is usually not so satisfactory. And the power consumption is too high for a battery supplied portable instrument. A 12bit/150MSPS flash ADC AD9627-150, for instant, the power consumption reaches to $820 \mathrm{~mW}$, whereas the SNR is only $69.2 \mathrm{~dB}$ at $150 \mathrm{MSPS}^{5)}$.

In recent large scale experiments, hundreds and thousands of input channels would be measured. For example, the inner detector of the Super Kamiokande detector in Japan contends 11146 PMTs $^{6}$. The energy information from each PMT channel should be readout. Using thousands of flash ADC to construct such a huge electronic system, cost, size, and reliability should be concerned. Instead of the Digital Pulse Processing (DPP)

*Corresponding Author, E-mail: linyc@cdut.edu.cn

(C) Atomic Energy Society of Japan technique, Charge to Time Conversion (QTC) is used in this splendid construction ${ }^{6}$. QTC technique is also used to measure the charge of particles in the drift chamber in the KEK B factory experiment BELLE ${ }^{7)}$.

As a pulse area sensitive circuit, QTC is expected to have better sensitivity, higher noise rejection rate and less waveform dependency, with simpler circuit configuration, lower cost and higher reliability. Since the QTC technique has such outstanding features, it is proposed to perform energy spectrum measurement tasks in radiation spectroscopy region.

\section{Simplified schematic of QTC}

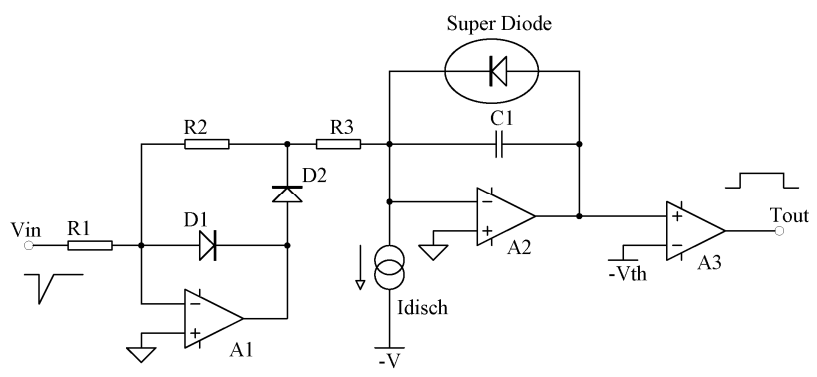

Fig. 1 Simplified schematic of the charge to time convertor

A simplified schematic of the charge to time convertor is designed and presented in Fig.1. The input negative pulse is rectified by an inverting active rectifier. The output of the rectifier charges $\mathrm{C} 1$ via $\mathrm{R} 3$. The charge fed into $\mathrm{C} 1$ is proportional to the area of the input pulse. A constant current source $\mathrm{I}_{\text {disch, }}$, will discharge $\mathrm{C} 1$. The discharge time interval Tout can be easily described as 


$$
\mathrm{T}_{\text {out }}=\frac{\mathrm{Q}}{\mathrm{I}_{\text {disch }}} .
$$

Where $\mathrm{Q}$ is the charge fed into $\mathrm{C} 1 . \mathrm{I}_{\text {disch }}$ is the discharge current. Because the $\mathrm{I}_{\text {disch }}$ is a constant current source, Tout is proportional to the charge. Therefore, Tout is proportional to the area of the input pulse. The width of the charge and discharge waveform is then converted into a logic signal by the discriminator A3.

Attention should be paid that the output voltage would be maintained at zero if the charging current through R3 is less than the discharge current $I_{\text {disch. }}$. To have a maximum dynamic range, the discharge current should be designed to be as small as possible. In this experiment, $\mathrm{I}_{\text {disch }}$ is set to be $25 \mu \mathrm{A}$.

An active rectifier based Super Diode crossing the inverting input and the output of the amplifier A2 provides a DC rout to prevent the discharge current from pulling the voltage out of the ground level.

The width of the QTC logical output can be easily converted into a digital value in the programmable logic device FPGA or CPLD. A CPLD EPM3128 with a $100 \mathrm{MHz}$ clock is used in the experiment. The maximum energy bin number is designed to be 8192 , which is corresponding to 4096 ns.

\section{Application in Gamma spectroscopy}

As a pulse area sensitive circuit, QTC is expected to have better sensitivity, higher noise rejection ratio and less waveform dependency. Interfacing to scintillation detectors with Photo Multiply Tube (PMT) needs neither pulse shaping circuit nor baseline restoration circuit. The voltage gain of the first stage of the QTC is set to be -1 , e.g., $\mathrm{R} 1=\mathrm{R} 2=1 \mathrm{k} \mathrm{ohm}$, and R3=200 ohm.

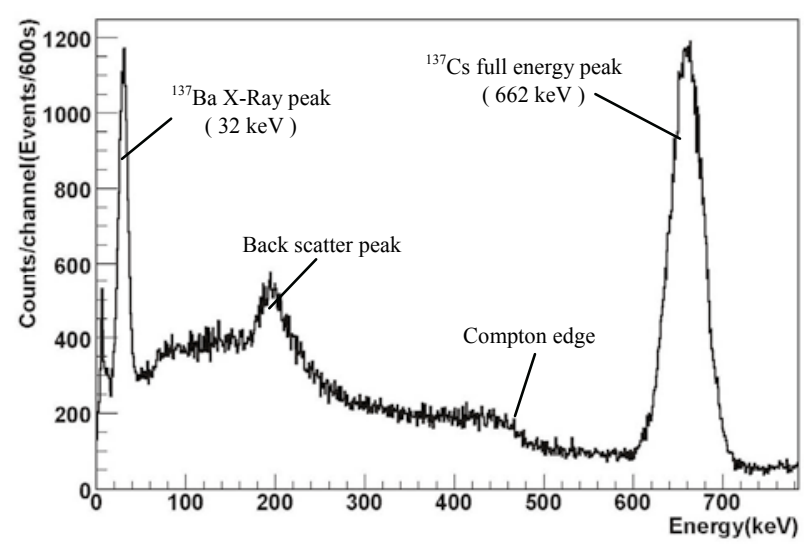

Fig.2 ${ }^{137} \mathrm{Cs}$ spectrum taken by $\Phi 75 \times 75 \quad \mathrm{~mm} \quad \mathrm{NaI}(\mathrm{Tl})$ scintillation detector connect with the QTC circuit without shaping and baseline restoration circuit

Figure 2 shows a ${ }^{137} \mathrm{Cs}$ gamma spectrum taken by a $\Phi 75 \times 75 \mathrm{~mm} \mathrm{NaI}(\mathrm{Tl})$ scintillation detector connect to the QTC circuit without any shaping and baseline restoration circuit. The detector is supplied by the Beijing nuclear instrument factory. A transistor preamplifier is installed inside the detector. From this figure, the ${ }^{137} \mathrm{Ba} \mathrm{X}$-ray peak at around $32 \mathrm{keV}$, the back scatter peak, the Compton edge, and the ${ }^{137} \mathrm{Cs}$ full energy peak at $662 \mathrm{keV}$ are clearly observed. The energy resolution (FWHM) is about $7.38 \%$ at $662 \mathrm{keV}$.

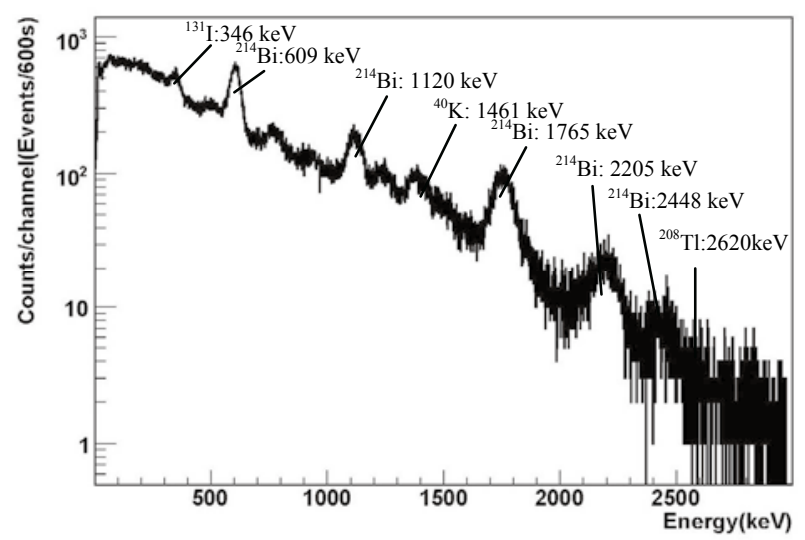

Fig. 3 Energy spectrum of a uranium ore sample

To evaluate the energy linearity, energy spectra of uranium ore sample and gamma background on the concrete ground are also measured. The spectrum of the uranium ore sample is shown in Fig.3 in log scale. The peak positions according to ${ }^{131} \mathrm{I},{ }^{214} \mathrm{Bi},{ }^{40} \mathrm{~K}$, and ${ }^{208} \mathrm{Tl}$ are manually read. The peak position for ${ }^{137} \mathrm{Cs}$ can be measured too. A linear fit to these points is performed, the energy nonlinear error NE can be calculated by

$$
\mathrm{NE}=\frac{\text { Calibration value-reference energy }}{\text { full energy rang }} \times 100 .
$$

The reference energy and the corresponding nuclide, peak positions, calibration results and the nonlinear error are listed in Table 1. From the table, the maximum nonlinear error is within $\pm 0.15 \%$.

Table 1 Energy calibration results and energy nonlinearity

\begin{tabular}{lllll}
\hline Nuclide & $\begin{array}{l}\text { Energy } \\
(\mathrm{keV})\end{array}$ & $\begin{array}{l}\text { Peak } \\
\text { position } \\
\text { (bin) }\end{array}$ & $\begin{array}{l}\text { Calibration } \\
\text { results } \\
(\mathrm{keV})\end{array}$ & $\begin{array}{l}\text { Nonlinear } \\
\text { error } \\
(\%)\end{array}$ \\
\hline${ }^{131} \mathrm{I}$ & 346 & 1015 & 348.6 & 0.09 \\
${ }^{214} \mathrm{Bi}$ & 609 & 1725 & 608.3 & -0.02 \\
${ }^{137} \mathrm{Cs}$ & 662 & 1865 & 659.5 & -0.08 \\
${ }^{214} \mathrm{Bi}$ & 1120 & 3118 & 1117.9 & -0.07 \\
${ }^{40} \mathrm{~K}$ & 1461 & 4055 & 1460.6 & 0.01 \\
${ }^{214} \mathrm{Bi}$ & 1765 & 4875 & 1760.6 & -0.15 \\
${ }^{214} \mathrm{Bi}$ & 2205 & 6090 & 2205.0 & 0.00 \\
${ }^{214} \mathrm{Bi}$ & 2448 & 6760 & 2450.1 & 0.07 \\
${ }^{208} \mathrm{Tl}$ & 2620 & 7220 & 2618.4 & 0.11 \\
\hline
\end{tabular}




\section{Application in Alpha spectrum based radon measurement}

Another application is to interface a silicon surface barrier (SSB) detector to the QTC without any charge sensitive amplifier. The first stage of the QTC circuit is configured into a current sensitive active rectifier. The application circuit is shown in Fig.4.

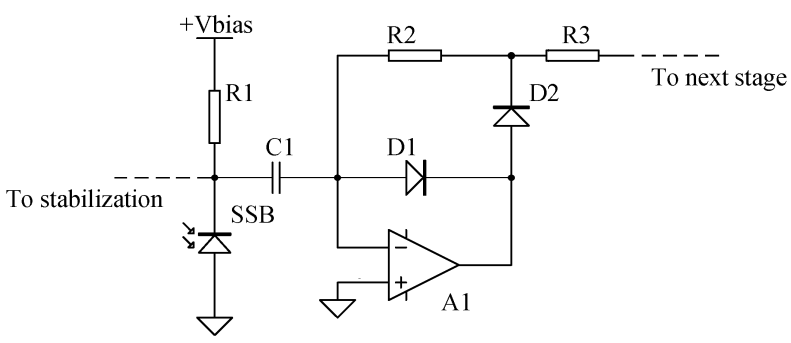

Fig.4 A SSB detector connect with the QTC

The input of the QTC circuit here is current sensitive. The corresponding current gain of the QTC input stage is $A_{I}=R 2 / R 3=1 \mathrm{Mohm} / 1 \mathrm{kohm}=1000$. Such a current sensitive front end circuit is not sensitive to any equivalent input capacitor. As a result, the length of the detector coaxial cable is not critical. In this experiment, it is large than $40 \mathrm{~cm}$. Another advantage is that the stabilization circuit can be connected to the anode of the detector. The stabilization circuit can make compensation to the drift of the detector leakage current.

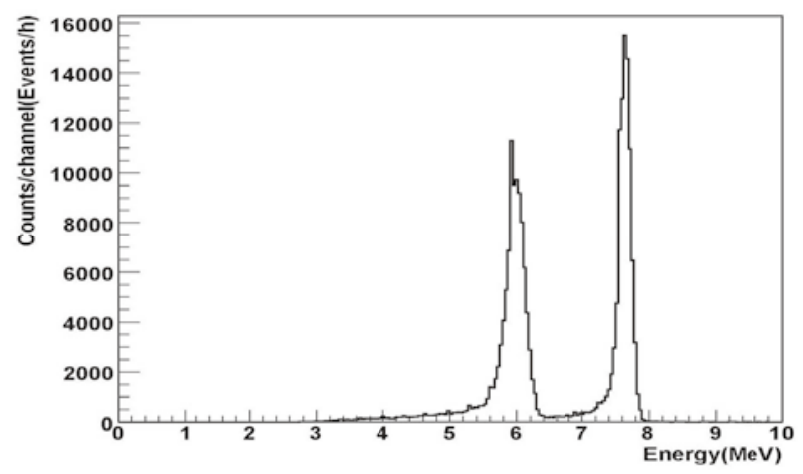

Fig.5 Alpha spectrum of radon daughters taken by SSB detector connect with the QTC without charge sensitive amplifier

Figure 5 shows an alpha spectrum taken from the experiment. The peaks at $6.00 \mathrm{MeV}$ and $7.69 \mathrm{MeV}$ are individually released from ${ }^{222} \mathrm{Rn}$ daughter nuclide ${ }^{218} \mathrm{Po}$ and ${ }^{214} \mathrm{Po}$. The corresponding energy resolution is about $204 \mathrm{keV}$ at $7.69 \mathrm{MeV}$. In the experiment, the SSB detector is installed at the bottom center of a cylinder chamber. A high voltage of about $-2 \mathrm{kV}$ is applied between the detector and the chamber. Most of the $5.45 \mathrm{MeV}$ alpha particles, emitted by ${ }^{222} \mathrm{Rn}$ directly in the chamber, react with air and cannot reach the detector. However, the decay products ${ }^{218} \mathrm{Po}$ are collected onto the surface of the SSB detector by the high voltage. This is why the $5.45 \mathrm{MeV}$ alpha particles are not observed in the spectrum.

The radon measurement system has been run for hundreds hours in a radon permeability through material measurement experiment. The experimental goal is to make shielding material selection for a low background reactor neutrino experiment ${ }^{8)}$. Accumulation readings were performed every hour. The alpha spectrum data were logged into a computer. To check the reliability of the whole experimental system, the $7.69 \mathrm{MeV}$ peak position at every $12 \mathrm{~h}$ is determined from a Gaussian fit. Figure 6 shows all the peak positions during the experiment for more than $200 \mathrm{~h}$. In the case of 1024 energy bin in total, the maximum peak drift is +3 bin and -5 bin.

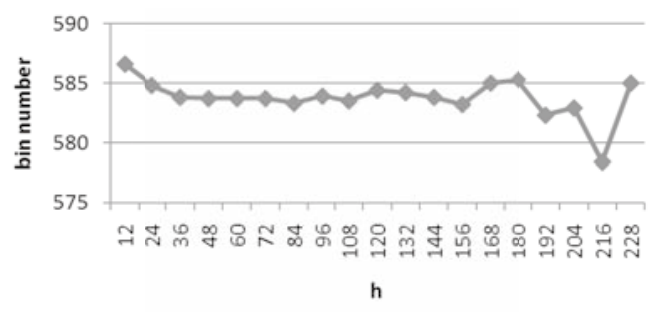

Fig.6 7.69 MeV Peak position during the Radon permeability through material measurement experiment

\section{Conclusion}

A Charge to Time converter is used to interface to the scintillation detector and the silicon surface barrier detector. With no shaping and baseline restoration circuit in use, the application circuit configuration becomes simple. Experimental results indicate that the performances, such as the linearity, energy resolution, dynamic range and reliability, etc, are satisfactory as expected. This QTC technique can be widely adopted in various applications of radiation spectroscopy.

\section{Acknowledgment}

This work has been supported by the National Natural Science Foundation of China (10890093) and the Chinese Post Doctor Science Foundation (20080430375).

\section{References}

1) S.W. Pauly. "Development of a shaping amplifier, gated integrator and $16 \mathrm{~K}$ Channel $\mathrm{ADC}$ for a portable spectroscopy system". Nucl. Instr. and Meth. A 422 379-384 (1999).

2) Dinh Sy Hien, Toshihiko Senzaki. "Development of a fast 12-bit ADC for a nuclear spectroscopy system". Nucl. Instr. and Meth. A 457 356-360(2001).

3) J. Walter, M. Barreiro, L. Sajo-Bohus, et.al. "New approach in add-on multi-channel analyzer for gamma ray spectrometry". Nucl. Instr. and Meth. A 545 776-783(2005).

4) W.K. Warburton, P.M. Grudberg. "Current trends in developing digital signal processing electronics for semiconductor 
detectors". Nucl. Instr. and Meth. A 568 350-358(2006).

5) Analog Devices, Inc. "12-Bit, 80 MSPS/105 MSPS/125 MSPS/150 MSPS, $1.8 \mathrm{~V}$ Dual Analog-to-Digital Converter AD9627”. Rev.A. www.analog.com(2007-2009).

6) The Super Kamiokande Collaboration, K. Fujita, Y. Gando, A. Hasegawa, et al. "The Super Kamiokande Detector". Nucl. Instr. and Meth. A 501 418-462(2003).
7) Y. Fujita, N. Ujiie, K. Yoshidab, et.al. "Test of charge-to-time conversion and multi-hit TDC technique for the BELLE CDC readout". Nucl. Instr. and Meth. A 405 105-110(1998).

8) Shaomin Chen, "The Daya Bay Reactor Neutrino Experiment". J. Phys.: Conf. Ser. 120 052024, doi:10.1088/1742-6596/120/5/052024(2008). 